\title{
Axial computed tomography of the skull base
}

The trigeminal nerve should have been accorded the roman numeral of CN V [not "CN VIII" as was published online] in answers "B" and "C" of this Endgames quiz (BMJ 2014;348:g2958, doi:10.1136/bmj.g2958).
Cite this as: BMJ 2014;348:g3200

๑ BMJ Publishing Group Ltd 2014 